\title{
ON THE DIRICHLET SPACE FOR FINITELY CONNECTED REGIONS
}

\author{
KIT CHAK CHAN
}

\begin{abstract}
This paper is devoted to the study of the Dirichlet space $\operatorname{Dir}(G)$ for finitely connected regions $G$; we are particularly interested in the algebra of bounded multiplication operators on this space. Results in different directions are obtained. One direction deals with the structure of closed subspaces invariant under all bounded multiplication operators. In particular, we show that each such subspace contains a bounded function. For regions with circular boundaries we prove that a finite codimensional closed subspace invariant under multiplication by $z$ must be invariant under all bounded multiplication operators, and furthermore it is of the form $p \operatorname{Dir}(G)$, where $p$ is a polynomial with all its roots lying in $G$. Another direction is to study cyclic and noncyclic vectors for the algebra of all bounded multiplication operators. Typical results are: if $f \in \operatorname{Dir}(G)$ and $f$ is bounded away from zero then $f$ is cyclic; on the other hand, if the zero set of the radial limit function of $f$ on the boundary has positive logarithmic capacity, then $f$ is not cyclic. Also, some other sufficient conditions for a function to be cyclic are given. Lastly, we study transitive operator algebras containing all bounded multiplication operators; we prove that they are dense in the algebra of all bounded operators in the strong operator topology.
\end{abstract}

\section{INTRODUCTION}

Let $G$ be a domain in the complex plane $\mathbb{C}$. The Bergman space $B(G)$ is the Hilbert space of functions $f$ analytic in $G$ such that $|f|^{2}$ is integrable with respect to the area measure $d A$ on $G$; the norm in this space is given by

$$
\|f\|_{B(G)}^{2}=\int_{G}|f|^{2} d A .
$$

The Dirichlet space $\operatorname{Dir}(G)$ for the domain $G$ is the Hilbert space of functions $f$ analytic in $G$ whose derivative $f^{\prime}$ lies in $B(G)$. Fixing a point $w$ in $G$, we define a norm for $\operatorname{Dir}(G)$ by

$$
\|f\|_{\operatorname{Dir}(G)}^{2}=|f(w)|^{2}+\int_{G}\left|f^{\prime}\right|^{2} d A=|f(w)|^{2}+\left\|f^{\prime}\right\|_{B(G)}^{2} .
$$

Received by the editors March 30, 1988 and, in revised form, September 1, 1988.

1980 Mathematics Subject Classification (1985 Revision). Primary 46E20, 47B38; Secondary 47A $15,30 \mathrm{H} 05$.

Key words and phrases. Dirichlet space, finitely connected region, multiplication operators, invariant subspaces, cyclic vectors, transitive operator algebras, logarithmic capacity.

This paper includes part of the author's dissertation written under Professor Allen Shields at the University of Michigan. The author wishes to thank Professor Allen Shields for his guidance and encouragement.

The research was supported in part by a grant from NSF. 
A function $f$ analytic in $G$ is in $\operatorname{Dir}(G)$ if and only if the area (counting multiplicity) of $f(G)$ is finite. Of course, the value of the norm depends on the fixed point $w$ in $G$. However, the norms obtained by fixing different points are equivalent; see, for example, Proposition 6 of [BrSh].

In case $G$ is the open unit disk $\mathbb{D}$ centered at the origin, the functions $f$ in $\operatorname{Dir}(\mathbb{D})$ have a power series representation; $f(z)=\sum_{n=0}^{\infty} a_{n} z^{n}$. The norm of $\operatorname{Dir}(\mathbb{D})$ can be taken as

$$
\|f\|_{\operatorname{Dir}(\mathbb{D})}^{2}=|f(0)|^{2}+\int_{\mathbb{D}}\left|f^{\prime}\right|^{2} d A=\left|a_{0}\right|^{2}+\pi \sum_{n=1}^{\infty} n\left|a_{n}\right|^{2} .
$$

If $f$ is in $\operatorname{Dir}(\mathbb{D})$, then $\sum\left|a_{n}\right|^{2}$ is finite and hence $f$ is also in the Hardy space $H^{2}$. In particular, the radial limit function of $f$ is defined almost everywhere on the boundary $\partial \mathbb{D}$ of $\mathbb{D}$. The polynomials are dense in $\operatorname{Dir}(\mathbb{D})$; in fact the partial sums of the series for $f$ converge to $f$ in the norm topology. One can also check that if $f \in \operatorname{Dir}(\mathbb{D})$ then $z f \in \operatorname{Dir}(\mathbb{D})$.

A function $\phi$ analytic in $G$ is said to be a multiplier for $\operatorname{Dir}(G)$ if $\phi \operatorname{Dir}(G)$ $\subset \operatorname{Dir}(G)$, that is $\phi f$ is in $\operatorname{Dir}(G)$ if $f$ is in $\operatorname{Dir}(G)$. By $M(\operatorname{Dir}(G))$, we denote the algebra of all multipliers for $\operatorname{Dir}(G)$. Every multiplier will automatically induce a linear transformation $M_{\phi}: \operatorname{Dir}(G) \rightarrow \operatorname{Dir}(G)$ defined by

$$
M_{\phi} f=\phi f, \quad \text { for all } f \text { in } \operatorname{Dir}(G) .
$$

One can show that $M_{\phi}$ is a bounded linear operator; this follows from the closed graph theorem and the continuity of the linear functionals of evaluation at points in $G$. We call $M_{\phi}$ a multiplication operator. Since $\operatorname{Dir}(G)$ contains constant functions, the multipliers are functions in $\operatorname{Dir}(G)$. Also we know that a multiplier is necessarily bounded in $G$ (see [DuRS, Lemma 11]). We have an inclusion relation: $M(\operatorname{Dir}(G)) \subset \operatorname{Dir}(G) \cap H^{\infty}(G)$, where $H^{\infty}(G)$ denotes the algebra of all bounded analytic functions in $G$. In case $\operatorname{Dir}(G) \subset B(G)$, every function analytic in the closure of $G$, denoted by $G^{-}$, is a multiplier for $\operatorname{Dir}(G)$.

We call a circular domain any domain that is obtained by removing a finite number of disjoint closed subdisks (some perhaps of radius zero) from the open unit disk. If $G$ is a (nonempty) finitely connected domain in $\mathbb{C}$, there exists a conformal mapping $\psi$ of $G$ onto some circular domain $\Omega$. We can choose $\psi$ so that the composition map $C_{\psi}: f \rightarrow f \circ \psi$ is an isometry from $\operatorname{Dir}(\Omega)$ onto $\operatorname{Dir}(G)$. We shall consider circular domains $\Omega$. Without loss of generality, we assume that each removed closed subdisk has positive radius because otherwise the removed disk is just a point and it is a removable singularity for all the functions in $\operatorname{Dir}(\Omega)$. Since $\operatorname{Dir}(G), M(\operatorname{Dir}(G)), H^{\infty}(G)$ are all preserved by conformal maps, those results using only these concepts are valid in arbitrary finitely connected regions. 
In this paper, we shall add and multiply functions defined on different domains. Without further comment, the domain of the sum or the product will be taken to be the intersection of the domains of the summands or the factors.

\section{Preliminaries}

In this section, we set forth some preliminaries that we shall need later. We begin by looking at the $\operatorname{Dirichlet}$ space $\operatorname{Dir}(G)$ for an open connected set $G$ in $\mathbb{C}$. It is easy to show that in case $G$ is bounded, $\operatorname{Dir}(G)$ is contained in $B(G)$ if and only if $z$ is a multiplier for $\operatorname{Dir}(G)$. There are some other equivalent conditions; see $[\mathrm{AxSh}]$. Consider the following property.

If $w \in G, f \in \operatorname{Dir}(G)$ and $f(w)=0$, then $f(z) /(z-w)$ is in $\operatorname{Dir}(G)$.

We remark that if $\operatorname{Dir}(G) \subset B(G)$, then $\operatorname{Dir}(G)$ has the above property. The converse is true when $G$ is bounded. These follow from simple computations. It is well known that if $z$ is a point in $G$ and $n \geq 0$ is an integer then the linear functional $\lambda_{z, n}: f \rightarrow f^{(n)}(z)$ is norm continuous on $\operatorname{Dir}(G)$.

Lemma 2.1. If $K$ is a compact subset of $G$ and $n \geq 0$ is an integer, then there exists a constant $C=C(n, K)>0$ such that for all $z$ in $K$ and all functions $f$ in $\operatorname{Dir}(G),\left|f^{(n)}(z)\right| \leq C\|f\|_{\operatorname{Dir}(G)}$.

Proof. Apply the principle of uniform boundedness to the family of bounded linear functionals $\lambda_{z, n}: f \rightarrow f^{(n)}(z)$, where $z$ is in $K$.

The analogue of this result is also true for Bergman spaces.

Lemma 2.2. If $K$ is a compact subset of $G$ and $n \geq 0$ is an integer, then there exists a constant $C=C(n, K)>0$ such that for all $z$ in $K$ and all functions $f$ in $B(G),\left|f^{(n)}(z)\right| \leq C\|f\|_{B(G)}$.

Fix $0<R<1$. From now on we shall use the following notation.

Notation. Denote $\mathscr{R}=\{z \in \mathbb{C}: R<|z|\}$ and $\mathscr{A}=\{z \in \mathbb{C}: R<|z|<1\}$.

The following two lemmas establish some basic facts about the Dirichlet spaces and the Bergman spaces for these two regions. The proofs follow from direct calculations using Laurent series, and they are omitted.

Lemma 2.3. Let $f(z)=\sum_{n=-\infty}^{+\infty} a_{n} z^{n}$ be a function analytic in $\mathscr{R}$. We have the following.

(1) If $f$ is in $\operatorname{Dir}(\mathscr{R})$, then $a_{n}=0$ for $n>0$ and

$$
\left\|f^{\prime}\right\|_{B(\cdot \Re)}^{2}=\pi \sum_{n=-\infty}^{-1}|n|\left|a_{n}\right|^{2} R^{2 n} .
$$

(2) If $f$ is in $B(\mathscr{R})$, then $a_{n}=0$ for $n \geq-1$ and

$$
\|f\|_{B(\mathscr{R})}^{2}=\pi \sum_{n=-\infty}^{-2}|n+1|^{-1}\left|a_{n}\right|^{2} R^{2(n+1)}
$$


(3) If $f$ is in $\operatorname{Dir}(\mathscr{R})$, then $f / z$ is also in $\operatorname{Dir}(\mathscr{R})$ and $f / z^{2}$ is in $B(\mathscr{R})$.

Lemma 2.4. Let $f(z)=\sum_{n=-\infty}^{+\infty} a_{n} z^{n}$ be a function analytic in $\mathscr{A}$. We have the following.

(1) If $f$ is in $\operatorname{Dir}(\mathscr{A})$, then

$$
\left\|f^{\prime}\right\|_{B(\mathscr{Q})}^{2}=\pi \sum_{n \neq 0} n\left|a_{n}\right|^{2}\left(1-R^{2 n}\right) .
$$

(2) If $f$ is in $\operatorname{Dir}(\mathscr{A})$, then $z f$ is in $\operatorname{Dir}(\mathscr{A}) .($ Equivalently, $\operatorname{Dir}(\mathscr{A}) \subset$ $B(\mathscr{A})$.)

(3) If $f$ is in $B(\mathscr{A})$, then

$$
\|f\|_{B(\mathscr{A})}^{2}=\pi \sum_{n \neq-1}(n+1)^{-1}\left|a_{n}\right|^{2}\left(1-R^{2(n+1)}\right)+2 \pi\left|a_{-1}\right|^{2} \log \left(R^{-1}\right) .
$$

Without further comment, we shall apply the results (with the corresponding modifications) in Lemmas 2.3 and 2.4 to the Dirichlet spaces for regions that can be obtained by translating, shrinking or enlarging $\mathscr{A}$ or $\mathscr{R}$.

We shall use the following definition throughout this paper.

Definition. Let $g(z)=\sum_{n=-\infty}^{+\infty} b_{n}(z-\zeta)^{n}$ be a Laurent series about the point $\zeta$. For $0<t<1$, we define $g_{t}(z)=\sum_{n=-\infty}^{+\infty} b_{n} t^{|n|}(z-\zeta)^{n}$.

In case $g(z)=\sum_{n=0}^{\infty} b_{n} z^{n}$ is an analytic function in $\mathbb{D}$, then $g_{t}(z)=g(t z)$ is a function analytic in $t^{-1} \mathbb{D}=\left\{z \in \mathbb{C}:|z|<t^{-1}\right\}$. If $g(z)=\sum_{n=-\infty}^{-1} b_{n} z^{n}$ is a function analytic in $\mathscr{R}$, then $g_{t}(z)=g\left(t^{-1} z\right)$ is a function analytic in $\{z \in$ $\mathbb{C}:|z|>t R\}$. Also, if $h(z)=\sum_{n=-\infty}^{+\infty} a_{n} z^{n}$ is analytic in $\mathscr{A}$, we can apply the Cauchy integral formula to write $h$ as $h=h_{0}+h_{1}$, where $h_{0}(z)=\sum_{n=0}^{\infty} a_{n} z^{n}$ is analytic in $\mathbb{D}$ and $h_{1}(z)=\sum_{n=-\infty}^{-1} a_{n} z^{n}$ is analytic in $\mathscr{R}$. By our definition, we have $h_{t}=\left(h_{0}\right)_{t}+\left(h_{1}\right)_{t}$, and so $h_{t}$ is analytic in $\left\{z \in \mathbb{C}: t R<|z|<t^{-1}\right\}$. Consequently, $h_{t} \in M(\operatorname{Dir}(\mathscr{A}))$. On the other hand it is easy to see, by using Lemma 2.3, that if $f$ is in $\operatorname{Dir}(\mathscr{R})$ then $f_{t}$ is in $\operatorname{Dir}(\mathscr{R})$. Using the previous lemmas, one can prove that if $f$ is a function in $\operatorname{Dir}(G)$ where $G$ denotes either $\mathbb{D}, \mathscr{R}$ or $\mathscr{A}$, then $f_{t} \rightarrow f$ in the norm topology as $t \uparrow 1$. This is also true if we replace $\operatorname{Dir}(G)$ by $B(G)$.

If $u(z)=\sum_{n=0}^{\infty} u_{n} z^{n}$ is a function analytic in $\mathbb{D}$, then $v(z)=u(R / z)=$ $\sum_{n=0}^{\infty} u_{n}(R / z)^{n}$ is a function analytic in $\mathscr{R}$. Furthermore, $u \in \operatorname{Dir}(\mathbb{D})$ if and only if $v \in \operatorname{Dir}(\mathscr{R})$, and also $u \in M(\operatorname{Dir}(\mathbb{D}))$ if and only if $v \in M(\operatorname{Dir}(\mathscr{R}))$. In fact $\left\|u^{\prime}\right\|_{B(\mathbb{D})}=\left\|v^{\prime}\right\|_{B(\mathscr{A})}$.

\section{SUM AND PRODUCT OF FUNCTIONS}

Let $G$ be an open connected set in $\mathbb{C}$. We use $H(G)$ to denote the algebra of all analytic functions in $G$ and $H^{\infty}(G)$ to denote the algebra of all bounded functions in $H(G)$. In case $G$ is unbounded, $H_{0}(G)$ denotes the space of all functions in $H(G)$ that vanish at $\infty$. 
Throughout this paper we shall use the following notation concerning a general finitely connected circular region.

Notation. Let $\Omega=\mathbb{D} \backslash\left(D_{1}^{-} \cup D_{2}^{-} \cup \cdots \cup D_{N}^{-}\right)$where $D_{i}^{-}=\left\{z:\left|z-\zeta_{i}\right| \leq r_{i}\right\}$ $(i=1, \ldots, N)$ are disjoint closed subdisks of the open unit disk $\mathbb{D}$. Fix a sufficiently small positive number $\eta$ so that $\eta<\inf \left\{\operatorname{dist}\left(\partial D_{i}, \partial D_{j}\right): 0 \leq\right.$ $i, j \leq N, i \neq j\}$ (where $D_{0}=\mathbb{D}$ ). Let $\Gamma_{0}=\left\{z:|z|=1-2^{-1} \eta\right\}$ and $\Gamma_{i}=$ $\left\{z:\left|z-\zeta_{i}\right|=r_{i}+2^{-1} \eta\right\} \quad(1 \leq i \leq N)$ be circles lying in $\Omega$ and concentric to the boundary circles of $\Omega$. By $\operatorname{Int}\left(\Gamma_{i}\right)$ and $\operatorname{Ext}\left(\Gamma_{i}\right)$ we denote the bounded and unbounded components of $\mathbb{C} \backslash \Gamma_{i}$ respectively. We let $A_{0}=\operatorname{Ext}\left(\Gamma_{0}\right) \cap \Omega$ and $A_{i}=\operatorname{Int}\left(\Gamma_{i}\right) \cap \Omega(i=1, \ldots, N)$. We remark that $A_{i}(i=0, \ldots, N)$ are annuli in $\Omega$. Finally, we denote $\Omega_{i}=\mathbb{C} \backslash D_{i}^{-}(i=1, \ldots, N)$.

An application of the Cauchy integral formula shows that if $f$ is in $H(\Omega)$, then we can write $f=f_{0}+f_{1}+\cdots+f_{N}$, where $f_{0} \in H(\mathbb{D})$ and $f_{i} \in H_{0}\left(\Omega_{i}\right)$. This is also true for bounded analytic functions; it is proved in [ChPS] that

$$
H^{\infty}(\Omega)=H^{\infty}(\mathbb{D})+H_{0}^{\infty}\left(\Omega_{1}\right)+\cdots+H_{0}^{\infty}\left(\Omega_{N}\right)
$$

where $H_{0}^{\infty}\left(\Omega_{i}\right)=H^{\infty}\left(\Omega_{i}\right) \cap H_{0}\left(\Omega_{i}\right)$. The next theorem tells us a similar decomposition phenomenon for the Dirichlet spaces.

Theorem 3.1. $\operatorname{Dir}(\Omega)=\operatorname{Dir}(\mathbb{D})+\operatorname{Dir}_{0}\left(\Omega_{1}\right)+\cdots+\operatorname{Dir}_{0}\left(\Omega_{N}\right)$, where $\operatorname{Dir}_{0}\left(\Omega_{i}\right)=$ $H_{0}\left(\Omega_{i}\right) \cap \operatorname{Dir}\left(\Omega_{i}\right)$.

Proof. For notational simplicity, we shall only prove the result for the case $N=$ 2. If $h$ is in $\operatorname{Dir}(\Omega)$, then $h=h_{0}+h_{1}+h_{2}$, where $h_{0} \in H(\mathbb{D}), h_{i} \in H_{0}\left(\Omega_{i}\right)$, $(i=1,2)$. We must prove that $h_{0} \in \operatorname{Dir}(\mathbb{D})$ and $h_{i} \in \operatorname{Dir}\left(\Omega_{i}\right)$.

To show that $h_{0}$ is in $\operatorname{Dir}(\mathbb{D})$, we prove that $\left|h_{0}^{\prime}\right|^{2}$ is integrable with respect to the area measure $d A$ on $A_{0}$ and $\mathbb{D} \backslash A_{0}$ separately. On $A_{0}$, both $h_{1}^{\prime}$ and $h_{2}^{\prime}$ are bounded and so they are square integrable. By our assumption $h^{\prime}$ is square integrable over $A_{0}$ and so is $h_{0}^{\prime}=h^{\prime}-h_{1}^{\prime}-h_{2}^{\prime}$. On the other hand, $h_{0}^{\prime}$ is bounded on $\mathbb{D} \backslash A_{0}$, it is square integrable.

We now show that $h_{1} \in \operatorname{Dir}\left(\Omega_{1}\right)$ and it will follow from the same argument that $h_{2} \in \operatorname{Dir}\left(\Omega_{2}\right)$. We write, for $z$ in $\Omega_{1}, h_{1}(z)=\sum_{n=1}^{\infty} a_{n}\left(z-\zeta_{1}\right)^{-n}$. So $h_{1}^{\prime}(z)=-\left(z-\zeta_{1}\right)^{-2} \sum_{n=1}^{\infty} n a_{n}\left(z-\zeta_{1}\right)^{-n+1}$. We denote $g(z)=-\left(z-\zeta_{1}\right)^{2} h_{1}^{\prime}(z)$. Since $\lim g(z)=a_{1}$ as $|z| \rightarrow \infty, g$ is bounded on $\Omega_{1} \backslash A_{1}$. Let $|g(z)|<M$ on $\Omega_{1} \backslash A_{1}$.

$$
\int_{\Omega_{1} \backslash A_{1}}\left|h_{1}^{\prime}\right|^{2} d A \leq M^{2} \iint_{\Omega_{1} \backslash A_{1}} r^{-3} d r d \theta, \quad \text { which is finite. }
$$

It remains to show that $h_{1}^{\prime}$ is square integrable over $A_{1}$. This is obvious because $h_{1}^{\prime}=h^{\prime}-h_{0}^{\prime}-h_{2}^{\prime}$, both $h_{0}^{\prime}$ and $h_{2}^{\prime}$ are bounded on $A_{1}$, and $h^{\prime}$ is square integrable over $A_{1}$.

Conversely, if $g_{0} \in \operatorname{Dir}(\mathbb{D})$ and $g_{i} \in \operatorname{Dir}\left(\Omega_{i}\right)(i=1,2)$ then $g_{0}^{\prime}, g_{1}^{\prime}, g_{2}^{\prime}$ are all square integrable over $\Omega$ and so is $g^{\prime}=g_{0}^{\prime}+g_{1}^{\prime}+g_{2}^{\prime}$. 
Not only is the decomposition theorem valid for the Dirichlet spaces, it is also valid for their algebras of multipliers.

Theorem 3.2. $M(\operatorname{Dir}(\Omega))=M(\operatorname{Dir}(\mathbb{D}))+M_{0}\left(\operatorname{Dir}\left(\Omega_{1}\right)\right)+\cdots+M_{0}\left(\operatorname{Dir}\left(\Omega_{N}\right)\right)$, where $M_{0}\left(\operatorname{Dir}\left(\Omega_{i}\right)\right)=H_{0}\left(\Omega_{i}\right) \cap M\left(\operatorname{Dir}\left(\Omega_{i}\right)\right)$.

Proof. We shall only prove the result for the case $N=2$. We first show that $M(\operatorname{Dir}(\Omega))$ is contained in the direct sum

Let $\phi \in M(\operatorname{Dir}(\Omega))$, so $\phi \in H^{\infty}(\Omega) \cap \operatorname{Dir}(\Omega)$. By Theorem 3.1, $\phi$ has a decomposition $\phi=\phi_{0}+\phi_{1}+\phi_{2}$, where $\phi_{0} \in \operatorname{Dir}(\mathbb{D})$ and $\phi_{i} \in \operatorname{Dir}\left(\Omega_{i}\right)$ $(i=1,2)$. We must show that $\phi_{0} \in M(\operatorname{Dir}(\mathbb{D}))$ and $\phi_{i} \in M\left(\operatorname{Dir}\left(\Omega_{i}\right)\right)$.

Let $f_{0} \in \operatorname{Dir}(\mathbb{D})$. We want to show that $\phi_{0} f_{0}$ is in $\operatorname{Dir}(\mathbb{D})$. Clearly its derivative $\left(\phi_{0} f_{0}\right)^{\prime}$ is square integrable over $\operatorname{Int}\left(\Gamma_{0}\right)$. To show that $\left(\phi_{0} f_{0}\right)^{\prime}$ is square integrable over $A_{0}$, we write $\left(\phi_{0} f_{0}\right)^{\prime}=\left(\phi f_{0}\right)^{\prime}-\left(\phi_{1} f_{0}\right)^{\prime}-\left(\phi_{2} f_{0}\right)^{\prime}$ and use the fact that $\operatorname{Dir}(\mathbb{D})$ is contained in $B(\mathbb{D})$.

Next we want to show that $\phi_{i} \in M\left(\operatorname{Dir}\left(\Omega_{i}\right)\right) \quad(i=1,2)$. It is enough to consider the case $i=1$. Let $f_{1} \in \operatorname{Dir}\left(\Omega_{1}\right)$. Note that $f_{1}^{\prime}$ is square integrable over $A_{1}$ and by Lemma $2.4, f_{1}$ is also square integrable over $A_{1}$, and so is $\left(\phi_{1} f_{1}\right)^{\prime}=\left(\phi f_{1}\right)^{\prime}-\left(\phi_{0} f_{1}\right)^{\prime}-\left(\phi_{2} f_{1}\right)^{\prime}$. We observe that $\left(\phi_{1} f_{1}\right)^{\prime}=$ $\phi_{1} f_{1}^{\prime}+\left(z-\zeta_{1}\right)^{2} \phi_{1}^{\prime}\left(z-\zeta_{1}\right)^{-2} f_{1}$. Clearly the first term on the right-hand side is square integrable over $\operatorname{Ext}\left(\Gamma_{1}\right)$. By Lemma 2.3, $\left(z-\zeta_{1}\right)^{-2} f_{1}$ is square integrable over $\operatorname{Ext}\left(\Gamma_{1}\right)$. Since $\phi_{1}$ vanishes at $\infty$, the Laurent series for $\phi_{1}$ at $\zeta_{1}$ has no constant term; one can show that $\left(z-\zeta_{1}\right)^{2} \phi_{1}^{\prime}(z)$ is bounded on $\operatorname{Ext}\left(\Gamma_{1}\right)$. Hence $\left(\phi_{1} f_{1}\right)^{\prime}$ is square integrable over $\operatorname{Ext}\left(\Gamma_{1}\right)$.

The converse can be proved by a similar method. We omit the details.

The next proposition establishes the fact that $\operatorname{Dir}(\Omega) \subset B(\Omega)$.

Proposition 3.3. The function $z$ is in $M(\operatorname{Dir}(\Omega))$.

Proof. Let $f \in \operatorname{Dir}(\Omega)$. Clearly $(z f)^{\prime}=z f^{\prime}+f$ is square integrable over $\Omega \backslash\left(A_{0} \cup \cdots \cup A_{N}\right)$. By Lemma 2.4 , it is also square integrable over $A_{0}, \ldots, A_{N}$.

The direct sum decomposition of functions $f$ in $H(\Omega)$ allows us to define $f_{t}(0<t<1)$ as an extension of our definition for Laurent series.

Definition. Let $f=f_{0}+f_{1}+\cdots+f_{N}$ be the unique decomposition of $f$ in $H(\Omega)$, where $f_{0} \in H(\mathbb{D})$ and $f_{i} \in H_{0}\left(\Omega_{i}\right)$. We define $f_{t}=\left(f_{0}\right)_{t}+\left(f_{1}\right)_{t}+\cdots+$ $\left(f_{N}\right)_{t}$.

Of course if $f \in \operatorname{Dir}(\Omega)$, then $f_{t} \rightarrow f$ in the norm as $t \uparrow 1$.

We shall use the following notation throughout the rest of this paper.

Notation. Fixing a point $w_{0}$ in $\Omega$, we define the norms for different Dirichlet spaces:

$$
\begin{array}{ll}
\|f\|^{2}=\left|f\left(w_{0}\right)\right|^{2}+\int_{\Omega}\left|f^{\prime}\right|^{2} d A & \text { for } f \text { in } \operatorname{Dir}(\Omega), \\
\|f\|_{0}^{2}=\left|f\left(w_{0}\right)\right|^{2}+\int_{\mathbb{D}}\left|f^{\prime}\right|^{2} d A & \text { for } f \text { in } \operatorname{Dir}(\mathbb{D}), \\
\|f\|_{i}^{2}=\left|f\left(w_{0}\right)\right|^{2}+\int_{\Omega_{i}}\left|f^{\prime}\right|^{2} d A & \text { for } f \text { in } \operatorname{Dir}\left(\Omega_{i}\right)(1 \leq i \leq N) .
\end{array}
$$


Proposition 3.4. There is a positive constant $C$ such that if $f_{0} \in \operatorname{Dir}(\mathbb{D}), f_{i} \in$ $\operatorname{Dir}\left(\Omega_{i}\right) \quad(1 \leq i \leq N)$ and $f=f_{0} f_{1} \cdots f_{N}$ is the product function, then $\|f\| \leq$ $C\left\|f_{0}\right\|_{0}\left\|f_{1}\right\|_{1} \cdots\left\|f_{N}\right\|_{N}$.

Proof. To estimate $\int_{\Omega}\left|f^{\prime}\right|^{2} d A$, we write $\Omega=K \cup A_{0} \cup A_{1} \cup \cdots \cup A_{N}$ where $K$ is a compact set, and estimate the integral over each subregion. One might use the fact that the linear map of multiplication by $\left(z-\zeta_{m}\right)^{-2}$ is a bounded operator from $\operatorname{Dir}\left(\Omega_{m}\right)$ to $B\left(\Omega_{m}\right)$ (see Lemma 2.3). We omit the details.

\section{CONVERGENCE PROPERTIES}

The main result in this section is the following: if $\phi \in \operatorname{Dir}(\Omega) \cap H^{\infty}(\Omega)$, $f \in \operatorname{Dir}(\Omega)$ and $\phi f \in \operatorname{Dir}(\Omega)$, then $\left\|\phi_{t} f-\phi f\right\| \rightarrow 0$ as $t \uparrow 1$. This result will be used repeatedly in the later sections. One corollary is that the operators of multiplication by rational functions with no poles in $\Omega^{-}$are dense in all multiplication operators in the strong operator topology (abbreviated SOT).

Lemma 4.1. For $f \in \operatorname{Dir}(\mathscr{A}), z \in \mathscr{A}$ and $0<t<1$, we have

$$
\left|f(z)-f_{t}(z)\right|^{2} \leq \frac{1}{\pi\left(1-R^{2}\right)}\left(\log \frac{1-t|z|}{1-|z|}+\log \frac{|z|-t R}{|z|-R}\right)\left\|f^{\prime}\right\|_{B(\mathscr{A})}^{2} .
$$

Proof. Let $f(z)=\sum_{n=-\infty}^{+\infty} a_{n} z^{n} \in \operatorname{Dir}(\mathscr{A})$. By the Cauchy-Schwarz inequality and Lemma 2.4 , we see that

$$
\begin{aligned}
\mid f(z) & -\left.f_{t}(z)\right|^{2} \leq\left(\sum_{n \neq 0}\left|a_{n}\right|^{2}|n|\left|1-R^{2 n}\right|\right) \\
& \times\left(\sum_{n \neq 0}|n|^{-1}\left|1-R^{2 n}\right|^{-1}\left(1-t^{|n|}\right)^{2}|z|^{2 n}\right) \\
\leq & \pi^{-1}\left\|f^{\prime}\right\|_{B(\mathscr{O})}^{2}\left(\sum_{n \neq 0}|n|^{-1}\left|1-R^{2 n}\right|^{-1}\left(1-t^{|n|}\right)^{2}|z|^{2 n}\right) .
\end{aligned}
$$

We first estimate the terms with positive powers.

$$
\begin{aligned}
& \sum_{n>0} n^{-1}\left(1-R^{2 n}\right)^{-1}\left(1-t^{n}\right)^{2}|z|^{2 n} \leq\left(1-R^{2}\right)^{-1} \sum_{n>0} n^{-1}\left(1-t^{n}\right)|z|^{n} \\
& =\left(1-R^{2}\right)^{-1}\{\log (1-|z| t)-\log (1-|z|)\} .
\end{aligned}
$$

Similarly we can show that

$$
\begin{aligned}
& \sum_{n<0}|n|^{-1}\left|1-R^{2 n}\right|^{-1}\left(1-t^{|n|}\right)^{2}|z|^{2 n} \\
& \quad \leq\left(1-R^{2}\right)^{-1}\{\log (|z|-R t)-\log (|z|-R)\} .
\end{aligned}
$$

Lemma 4.2. For $f \in \operatorname{Dir}(\mathscr{A}), R^{1 / 2}<t<1$ and $R t^{-1} \leq|z| \leq t$, we have

$$
\left|f(z)-f_{t}(z)\right|^{2} \leq \pi^{-1}\left(1-R^{2}\right)^{-1}\left(\log 2+\log \left(1+R^{-1 / 2}\right)\right)\left\|f^{\prime}\right\|_{B(\mathscr{\theta})}^{2} .
$$


Proof. The maximum value of $\left|f(z)-f_{t}(z)\right|^{2}$ is attained on the boundary.

By Theorem 3.1 (with $\Omega=\mathscr{A}$ ), every function $h$ in $\operatorname{Dir}(\mathscr{A}$ ) can be written as $h=h_{0}+h_{1}$, where $h_{0} \in \operatorname{Dir}(\mathbb{D})$ and $h_{1} \in \operatorname{Dir}_{0}(\mathscr{R})$. Furthermore there exist positive constants $C_{0}$ and $C_{1}$ depending on $R$ such that

$$
\left\|\left(h_{0}\right)^{\prime}\right\|_{B(\mathbb{D})} \leq C_{0}\left\|h^{\prime}\right\|_{B(\mathscr{A})}, \quad \text { and } \quad\left\|\left(h_{1}\right)^{\prime}\right\|_{B(\mathscr{R})} \leq C_{1}\left\|h^{\prime}\right\|_{B(\mathscr{A})} .
$$

Lemma 4.3. There exists a positive constant $C$ depending on $R$ such that if $f$, $g$ are in $\operatorname{Dir}(\mathscr{A})$ and $R^{1 / 2}<t<1$, then

$$
\int_{\mathscr{O}}\left|f-f_{t}\right|^{2}\left|\left(g_{t}\right)^{\prime}\right|^{2} d A<C\left\|f^{\prime}\right\|_{B(\mathscr{W})}^{2}\left\|g^{\prime}\right\|_{B(\mathscr{O})}^{2} \text {. }
$$

Proof. Let $f(z)=\sum_{n=-\infty}^{+\infty} a_{n} z^{n}$ and $g(z)=\sum_{n=-\infty}^{+\infty} b_{n} z^{n}$ be functions in $\operatorname{Dir}(\mathscr{A})$ and $R^{1 / 2}<t<1$ (so, $\left.R<R / t<t<1\right)$.

Throughout this proof, $C$ will denote a constant which depends only on $R$, however it may represent different values as the proof goes along. We can rewrite the left-hand side of the inequality in the statement of the lemma as

$$
\begin{aligned}
\int_{0}^{2 \pi} & \int_{R}^{1}\left|f-f_{t}\right|^{2}\left|\left(g_{t}\right)^{\prime}\right|^{2} r d r d \theta \\
& =\left(\int_{0}^{2 \pi} \int_{R}^{R / t}+\int_{0}^{2 \pi} \int_{R / t}^{t}+\int_{0}^{2 \pi} \int_{t}^{1}\right)\left|f-f_{t}\right|^{2}\left|\left(g_{t}\right)^{\prime}\right|^{2} r d r d \theta .
\end{aligned}
$$

It follows from Lemma 4.2 that the second summand above is bounded by an expression in the form of the right-hand side of the inequality. Next we show that the same is true for the third summand.

Let $g_{0}(z)=\sum_{n=0}^{\infty} b_{n} z^{n}$ and $g_{1}(z)=\sum_{n=-\infty}^{-1} b_{n} z^{n}$, and for $R<r<1$, let

$$
a(r)=\int_{0}^{2 \pi}\left|\left(g_{0}\right)^{\prime}\left(r e^{i \theta}\right)\right|^{2} d \theta=2 \pi \sum_{n=1}^{\infty}\left|b_{n}\right|^{2} n^{2} r^{2(n-1)} .
$$

Then $a(r)$ is an increasing function of $r$ and so,

$$
a(r) \int_{r}^{1} s d s \leq \int_{r}^{1} a(s) s d s=\int_{r}^{1} \int_{0}^{2 \pi}\left|\left(g_{0}\right)^{\prime}\left(s e^{i \theta}\right)\right|^{2} s d \theta d s .
$$

Thus, if $R<r<1$, then $a(r)(1-r)(1+r) / 2 \leq\left\|\left(g_{0}\right)^{\prime}\right\|_{B(. \mathscr{L})}^{2}$. Hence,

$$
\int_{0}^{2 \pi}\left|\left[\left(g_{0}\right)_{t}\right]^{\prime}\left(r e^{i \theta}\right)\right|^{2} d \theta \leq 2 C_{0}^{2}(1-r t)^{-1}\left\|g^{\prime}\right\|_{B(\mathscr{\prime})}^{2} .
$$

On the other hand, by Lemma 2.2,

$$
\operatorname{Max}\left\{\left|\left[\left(g_{1}\right)_{l}\right]^{\prime}(z)\right|^{2}: t \leq|z| \leq 1\right\} \leq C_{1}^{2} C\left\|g^{\prime}\right\|_{B(\mathscr{S})}^{2} .
$$

Hence,

$$
\begin{aligned}
\int_{0}^{2 \pi} & \left|\left(g_{t}\right)^{\prime}\left(r e^{i \theta}\right)\right|^{2} d \theta \\
\quad & =\int_{0}^{2 \pi}\left|\left[\left(g_{0}\right)_{t}\right]^{\prime}\left(r e^{i \theta}\right)\right|^{2} d \theta+\int_{0}^{2 \pi}\left|\left[\left(g_{1}\right)_{t}\right]^{\prime}\left(r e^{i \theta}\right)\right|^{2} d \theta \\
& \leq C(1-r t)^{-1}\left\|g^{\prime}\right\|_{B(\mathscr{O})}^{2} .
\end{aligned}
$$


Together with Lemma 4.1, this implies that

$$
\begin{aligned}
& \int_{0}^{2 \pi} \int_{t}^{1}\left|f-f_{t}\right|^{2}\left|\left(g_{t}\right)^{\prime}\right|^{2} r d r d \theta \\
& \leq C\left\|f^{\prime}\right\|_{B(\mathscr{A})}^{2}\left\|g^{\prime}\right\|_{B(\mathscr{A})}^{2}\left\{\int_{t}^{1}(1-r t)^{-1} \log \left[(1-r t)(1-r)^{-1}\right] d r\right. \\
&\left.\quad+\int_{t}^{1}(1-r t)^{-1} \log \left[(r-t R)(r-R)^{-1}\right] d r\right\} .
\end{aligned}
$$

It remains to show that the last two integrals are bounded by a constant which depends on $R$ only. The first one is bounded by $2 \log 2$ (see [Shie, Lemma 3]). For the second one, we can make trivial estimations.

For the first summand of the equation (4.1), we let

$$
b(r)=\int_{0}^{2 \pi}\left|\left(g_{1}\right)^{\prime}\left(r e^{i \theta}\right)\right|^{2} d \theta=2 \pi \sum_{n=-\infty}^{-1}\left|b_{n}\right|^{2} n^{2} r^{2(n-1)},
$$

and we proceed similarly. We leave the details to the readers.

Lemma 4.4. Suppose $f, g \in \operatorname{Dir}(\mathscr{A})$, then

$$
\lim \int_{\mathscr{A}}\left|f-f_{t}\right|^{2}\left|\left(g_{t}\right)^{\prime}\right|^{2} d A=0 \text { as } t \uparrow 1 \text {. }
$$

Proof. We first note that the lemma is true if $f$ is a polynomial in $z$ and $1 / z$. This is because $f_{t} \rightarrow f$ uniformly on the closure of $\mathscr{A}$.

Let $\varepsilon>0$, we choose a polynomial $p=p\left(z, z^{-1}\right)$ (a polynomial in $z$ and $z^{-1}$ ) such that $\left\|f^{\prime}-p^{\prime}\right\|_{B(\mathscr{A})}<\varepsilon$. Let $h=f-p$. By the triangle inequality,

$$
\left\|\left(f-f_{t}\right)\left(g_{t}\right)^{\prime}\right\|_{B(\mathscr{A})} \leq\left\|\left(p-p_{t}\right)\left(g_{t}\right)^{\prime}\right\|_{B(\mathscr{A})}+\left\|\left(h-h_{t}\right)\left(g_{t}\right)^{\prime}\right\|_{B(\mathscr{A})} .
$$

Now our result follows from Lemma 4.3.

Lemma 4.5. If $g \in \operatorname{Dir}(\mathbb{D})$ and $h \in \operatorname{Dir}(\mathscr{R})$, then

$$
\lim \left\|\left[(g h)_{t}-g_{t} h_{t}\right]^{\prime}\right\|_{B(\mathscr{A})}=0 \quad \text { as } t \uparrow 1 \text {. }
$$

Proof. By the triangle inequality, we have

$$
\begin{aligned}
& \left\|\left[(g h)_{t}\right]^{\prime}-\left[g_{t} h_{t}\right]^{\prime}\right\|_{B(\mathscr{A})} \leq\left\|\left[(g h)_{t}\right]^{\prime}-[g h]^{\prime}\right\|_{B(\mathscr{A})} \\
& +\left\|[g h]^{\prime}-\left[g_{t} h\right]^{\prime}\right\|_{B(\mathscr{A})}+\left\|\left[g_{t} h\right]^{\prime}-\left[g_{t} h_{t}\right]^{\prime}\right\|_{B(\mathscr{A})} .
\end{aligned}
$$

Clearly the first summand goes to zero as $t \uparrow 1$. The third summand is bounded by $\left\|\left(g_{t}\right)^{\prime}\left(h-h_{t}\right)\right\|_{B(\mathscr{A})}+\left\|g_{t}\left(h-h_{t}\right)^{\prime}\right\|_{B(\mathscr{A})}$. By Lemma 4.4, $\left\|\left(g_{t}\right)^{\prime}\left(h-h_{t}\right)\right\|_{B(\mathscr{A})} \rightarrow 0$ as $t \uparrow 1$. For the second term, we note that $g_{t}(z)$ is bounded on $R<|z|<$ $(1+R) / 2$ and apply Lemma 2.1 to $h(z)-h_{t}(z)$ on $(1+R) / 2<|z|<1$.

The second summand can be shown to go to zero by a similar method.

If $f, g$ are in $\operatorname{Dir}(\mathscr{A})$, then we can write $f=f_{0}+f_{1}, g=g_{0}+g_{1}$ where $f_{0}, g_{0}$ are in $\operatorname{Dir}(\mathbb{D})$ and $f_{1}, g_{1}$ are in $\operatorname{Dir}(\mathscr{R})$. Notice that

$$
(f g)_{t}-f_{t} g_{t}=\left(f_{0} g_{1}\right)_{t}+\left(f_{1} g_{0}\right)_{t}-\left(f_{0}\right)_{t}\left(g_{1}\right)_{t}-\left(f_{1}\right)_{t}\left(g_{0}\right)_{t} \text {. }
$$


The following lemma will be generalized in Theorem 4.7. However it is a useful tool in proving that.

Lemma 4.6. Suppose $g \in H^{\infty}(\mathscr{A}) \cap \operatorname{Dir}(\mathscr{A}), f \in \operatorname{Dir}(\mathscr{A})$ and $g f \in \operatorname{Dir}(\mathscr{A})$. Then

$$
\left\|\left[g_{t} f-g f\right]^{\prime}\right\|_{B(\mathscr{O})} \rightarrow 0 \quad \text { as } t \uparrow 1 \text {. }
$$

Proof. Applying the triangle inequality, we have

$$
\begin{aligned}
& \left\|\left[g_{t} f-g f\right]^{\prime}\right\|_{B(\mathscr{O})} \leq\left\|\left[g_{t}\left(f-f_{t}\right)\right]^{\prime}\right\|_{B(\mathscr{W})} \\
& \quad+\left\|\left[g_{t} f_{t}-(g f)_{t}\right]^{\prime}\right\|_{B(\mathscr{W})}+\left\|\left[(g f)_{t}-g f\right]^{\prime}\right\|_{B(\mathscr{G})} .
\end{aligned}
$$

It is clear that the first and third summand go to zero as $t \uparrow 1$. For the second summand, we can use (4.2) and Lemma 4.5.

Suppose $X$ is a Banach space and $T$ is a bounded linear operator on $X$. A closed subspace $M$ of $X$ is said to be an invariant subspace for $T$ if $T M \subseteq M$. For $g \in \operatorname{Dir}(\Omega)$, we let $[g]$ denote the smallest closed subspace containing $g$ and invariant under $M_{z}, M_{1 /\left(z-\zeta_{1}\right)}, \ldots, M_{1 /\left(z-\zeta_{N}\right)}$. If $\psi$ is a function analytic in $\Omega^{-}$then it is a uniform limit of polynomials in $z,\left(z-\zeta_{1}\right)^{-1}, \ldots,\left(z-\zeta_{N}\right)^{-1}$. So $\psi f \in[f]$ if $f \in \operatorname{Dir}(\Omega)$.

Theorem 4.7. If $\phi \in H^{\infty}(\Omega) \cap \operatorname{Dir}(\Omega), f \in \operatorname{Dir}(\Omega)$ and $\phi f \in \operatorname{Dir}(\Omega)$, then $\left\|\phi_{t} f-\phi f\right\| \rightarrow 0$ as $t \uparrow 1$, and $\phi f \in[f]$.

Proof. We must show that

$$
\int_{\Omega}\left|\left(\phi_{t} f-\phi f\right)^{\prime}\right|^{2} d A \rightarrow 0 \quad \text { as } t \uparrow 1 .
$$

We write $\Omega=K \cup A_{0} \cup \cdots \cup A_{N}$ where $K$ is a compact set. It follows from Lemma 2.1 that the integral over $K$ goes to zero as $t \uparrow 1$. For the integrals over the annuli, one might use the decomposition $\phi=\phi_{0}+\phi_{1}+\cdots+\phi_{N}$ where $\phi_{0} \in H^{\infty}(\mathbb{D}) \cap \operatorname{Dir}(\mathbb{D})$ and $\phi_{i} \in H_{0}^{\infty}\left(\Omega_{i}\right) \cap \operatorname{Dir}\left(\Omega_{i}\right) \quad(1 \leq i \leq N)$. Now the theorem is an easy consequence of Lemma 4.6.

Theorem 4.8. $\left\{M_{\phi}: \phi \in M(\operatorname{Dir}(\Omega))\right\}=S O T$-closure of $\left\{M_{r}: r \in \mathscr{G}\right\}$, where $\mathscr{G}$ is the set of all rational functions with poles only at $\zeta_{1}, \ldots, \zeta_{N}$.

Proof. Let $\left\{r_{\alpha}\right\} \subset \mathscr{G}$ be a net such that $M_{r_{n}} \rightarrow T$ (SOT) where $T$ is a bounded operator on $\operatorname{Dir}(\Omega)$. Let $\psi=T 1$, where we use 1 to denote the constant function of value 1 . So for all $z \in \Omega,\left|r_{\alpha}(z)-\psi(z)\right| \rightarrow 0$ and similarly if $f \in \operatorname{Dir}(\Omega),\left|r_{\alpha}(z) f(z)-(T f)(z)\right| \rightarrow 0$. Hence $(T f)(z)=(\psi f)(z)$ in $\Omega$.

The converse is a corollary of last theorem.

\section{CYCLIC VECTORS}

A vector in a Banach space is said to be a cyclic vector for an algebra of (bounded linear) operators on the space if the only closed subspace containing the vector and invariant under all operators in the algebra is the whole space. 
If the algebra is generated by a single operator, then the vector is said to be a cyclic vector for that operator. In what follows, we say that a function $g \in$ $\operatorname{Dir}(\mathscr{A})$ or $g \in \operatorname{Dir}(\Omega)$ is a cyclic vector when it is a cyclic vector for the corresponding algebra of all multiplication operators. Also by $\mathscr{P}_{k}(k \geq 1)$ we denote the set of all complex polynomials of $k$ variables, and by $\mathscr{P}$ we denote $\left\{p\left(z,\left(z-\zeta_{1}\right)^{-1}, \ldots,\left(z-\zeta_{N}\right)^{-1}\right): p\right.$ is in $\left.\mathscr{P}_{N+1}\right\}$.

It is clear that $g \in \operatorname{Dir}(\Omega)$ is cyclic if and only if $[g]=\operatorname{Dir}(\Omega)$ and that $\mathscr{P}$ is dense in $\operatorname{Dir}(\Omega)$. We remark the following.

Remarks. For $p \in \mathscr{P}$ and $f, g \in \operatorname{Dir}(\Omega)$,

(i) $p[f] \subset[f]$.

(ii) If $g \in[f]$, then $[g] \subset[f]$.

(iii) If $g \in[f]$ and $[g]=\operatorname{Dir}(\Omega)$, then $[f]=\operatorname{Dir}(\Omega)$.

(iv) $[f]=\operatorname{Dir}(\Omega)$ if and only if $1 \in[f]$.

(v) $[f]=\operatorname{Dir}(\Omega)$ if and only if there exists a sequence $\left\{p_{k}\right\}$ in $\mathscr{P}$ such that $p_{k} f \rightarrow 1$ in norm.

(vi) $[f]=\operatorname{Dir}(\Omega)$ if and only if there exists a sequence $\left\{p_{k}\right\}$ in $\mathscr{P}$ such that $p_{k} f \rightarrow 1$ weakly.

(vii) $[f]=\operatorname{Dir}(\Omega)$ if and only if there exists a sequence $\left\{p_{k}\right\}$ in $\mathscr{P}$ such that $p_{k}(z) f(z) \rightarrow 1$ for all $z$ in $\Omega$, and $\left\|p_{k} f\right\| \leq$ constant .

We remark that (i) follows directly from the fact that $p \in M(\operatorname{Dir}(\Omega))$. It is clear that (i) implies (ii) and (ii) implies (iii). Since $\mathscr{P}$ is dense in $\operatorname{Dir}(\Omega)$, [1] = $\operatorname{Dir}(\Omega)$ and now (iv), (v) and (vi) follows from (iii). Lastly (vii) is a straightforward application of a corollary in [BrSh, p. 272].

If a function $f$ in $\operatorname{Dir}(\Omega)$ vanishes at a point $z_{0}$ in $\Omega$, then $[f] \neq \operatorname{Dir}(\Omega)$. On the other hand, if $|f|$ is bounded below in some sense then $f$ has a chance to be cyclic. The analogue of next theorem for the case of the unit disk is contained in [BrSh]. With slight modifications, the proofs there also work for our situation and they are omitted.

Theorem 5.1. Let $f \in \operatorname{Dir}(\Omega), g \in H^{\infty}(\Omega) \cap \operatorname{Dir}(\Omega)$, and $\phi \in M(\operatorname{Dir}(\Omega))$. We have the following.

(1) $\phi f$ is cyclic if and only if both $\phi$ and $f$ are cyclic.

(2) If $|g(z)| \leq|f(z)|$ in $\Omega$ and $g^{2}$ is cyclic then $f$ is cyclic.

(3) If $|\phi(z)| \leq|f(z)|$ in $\Omega$ and $\phi$ is cyclic then $f$ is cyclic.

(4) If $g^{2}$ is cyclic then $g$ is cyclic.

(5) If $f \in H^{\infty}(\Omega) \cap \operatorname{Dir}(\Omega)$ and $f g$ is cyclic then $f$ and $g$ are both cyclic.

Corollary 5.2. If $f \in \operatorname{Dir}(\Omega)$ and there exists a positive constant $C$ such that $|f(z)|>C$ in $\Omega$, then $f$ is cyclic.

Proof. Take $\phi(z)=C$ in the result (3) of Theorem 5.1.

We know that $\operatorname{Dir}(\mathbb{D})$ and $\operatorname{Dir}(\Omega)$ are both closed under multiplication by $z$. In order to indicate the underlying Hilbert space that a multiplication operator is acting, we write $\left(M_{z}, \operatorname{Dir}(\mathbb{D})\right)$ and $\left(M_{z}, \operatorname{Dir}(\Omega)\right)$ to distinguish the two 
operators of multiplication by $z$ acting on $\operatorname{Dir}(\mathbb{D})$ and $\operatorname{Dir}(\Omega)$ respectively. Similar notation will be used for other multiplication operators.

We have shown that if $f_{0} \in \operatorname{Dir}(\mathbb{D}), f_{i} \in \operatorname{Dir}\left(\Omega_{i}\right)$, then the product function $f=f_{0} f_{1} \cdots f_{N}$ is in $\operatorname{Dir}(\Omega)$. The proposition that we are about to prove establishes the fact that the product of cyclic vectors is again cyclic.

Proposition 5.3. Suppose $f_{0} \in \operatorname{Dir}(\mathbb{D})$ and $f_{i} \in \operatorname{Dir}\left(\Omega_{i}\right) \quad(1 \leq i \leq N)$. If $f_{0}$ is cyclic for $\left(M_{z}, \operatorname{Dir}(\mathbb{D})\right)$ and each $f_{i}$ is cyclic for $\left(M_{1 /\left(z-\zeta_{i}\right)}, \operatorname{Dir}\left(\Omega_{i}\right)\right)$ $(1 \leq i \leq N)$, then the product function $f=f_{0} f_{1} \cdots f_{N}$ is cyclic.

Proof. Let $p_{0}$ be a polynomial in $z$ and $p_{i}$ be a polynomial in $\left(z-\zeta_{i}\right)^{-1}$ $(1 \leq i \leq N)$. So $p_{0} \in M(\operatorname{Dir}(\mathbb{D}))$, and $P_{i} \in M\left(\operatorname{Dir}\left(\Omega_{i}\right)\right) \quad(1 \leq i \leq N)$. We let $\eta_{i}$ be the norm of the operator of multiplication by $M_{p_{i}}$ on the corresponding Dirichlet space $(0 \leq i \leq N)$. By repeated applications of Proposition 3.4 (for different finitely connected regions), there exist positive constants $C_{1}, C_{2}, \ldots, C_{N}$ independent of $p_{i}, f_{i}$ such that

$$
\begin{aligned}
& \|p f-1\| \leq C_{N} \eta_{N-1} \cdots \eta_{0}\left\|f_{N-1}\right\|_{N-1} \cdots\left\|f_{0}\right\|_{0}\left\|p_{N} f_{N}-1\right\| \\
& \quad+C_{N-1} \eta_{N-2} \cdots \eta_{0}\left\|f_{N-2}\right\|_{N-2} \cdots\left\|f_{0}\right\|_{0}\left\|p_{N-1} f_{N-1}-1\right\|+\cdots+\left\|p_{0} f_{0}-1\right\| .
\end{aligned}
$$

We can choose $p_{0}, \ldots, p_{N}$ so that the right-hand side of the inequality above is as small as we like. Our result follows from Remark (iv).

We have known, from Corollary 5.2, that if $g$ is analytic and nonvanishing on $\Omega^{-}$, then $g$ is cyclic. Up to now we do not know what happens if $g$ does vanish at some points on the boundary. We remark that $u(z) \in \operatorname{Dir}(\mathbb{D})$ is cyclic for $\left(M_{z}, \operatorname{Dir}(\mathbb{D})\right)$ if and only if $v(z)=u(R / z)$ is cyclic for $\left(M_{1 / z}, \operatorname{Dir}(\mathscr{R})\right)$.

Lemma 5.4. If $p=p(z)$ is a polynomial in $z$ and $p$ has no roots in $\Omega$, then $p$ is cyclic.

Proof. Factorize $p=p_{0} p_{1} \cdots p_{N}$ where $p_{i}$ is a polynomial in $z, p_{0}$ has all its roots lying in $\mathbb{C} \backslash \mathbb{D}$ and $p_{i}$ has all its roots lying in $D_{i}^{-}$. We know that $p_{0}$ is cyclic for $\left(M_{z}, \operatorname{Dir}(\mathbb{D})\right)$ (see [BrSh, p. 289]).

Fix $N \geq i \geq 1$. We may assume $p_{i}$ is monic and let $p_{i}(z)=\left(z-a_{1}\right) \cdots(z-$ $\left.a_{k}\right)$ where $\left|a_{j}-\zeta_{i}\right| \leq r_{i} \quad(1 \leq j \leq k)$. Let $b_{j}=a_{j}-\zeta_{i}(1 \leq j \leq k)$.

The polynomial $s(z)=\left(r_{i}-b_{1} z\right) \cdots\left(r_{i}-b_{k} z\right)$ has no roots lying in $\mathbb{D}$, and hence it is cyclic for $\left(M_{z}, \operatorname{Dir}(\mathbb{D})\right)$. Since $s\left(r_{i} /\left(z-\zeta_{i}\right)\right)=r_{i}^{k} M_{1 /\left(z-\zeta_{i}\right)}^{k} p_{i}$ and so, $p_{i}$ is cyclic for $\left(M_{1 /\left(z-\zeta_{i}\right)}, \operatorname{Dir}\left(\Omega_{i}\right)\right)$. Our lemma is now an easy consequence of the last proposition.

Theorem 5.5. If $f$ is analytic on $\Omega^{-}$and it has no zeros in $\Omega$, then $f$ is cyclic. Proof. We have $f=p g$, where $p$ is a polynomial with no zeros in $\Omega$ and $|g|$ is bounded away from zero in $\Omega$. The polynomial $p$ is a multiplier and it is also cyclic. The result follows from Theorem 5.1 and Corollary 5.2.

For $f$ in $\operatorname{Dir}(\Omega)$, the following limits exist almost everywhere:

$$
\lim _{r \uparrow 1} f\left(r e^{i \theta}\right), \quad \lim _{r \downarrow r_{k}} f\left(\zeta_{k}+r e^{i \theta}\right) \quad(1 \leq k \leq N) .
$$


We define the following zero sets of radial limits for a function $f$ in $\operatorname{Dir}(\Omega)$.

$$
\begin{gathered}
Z_{0}(f)=\left\{e^{i \theta}: \lim f\left(r e^{i \theta}\right)=0 \text { as } r \uparrow 1\right\}, \\
Z_{k}(f)=\left\{e^{i \theta}: \lim f\left(\zeta_{k}+r e^{i \theta}\right)=0 \text { as } r \downarrow r_{k}\right\} \quad(1 \leq k \leq N) .
\end{gathered}
$$

We shall prove that if any one of these sets has positive logarithmic capacity, then $f$ cannot by cyclic. This result can be viewed as a partial converse of Corollary 5.2. However, it is not known whether $f$ is cyclic if none of these zero sets has positive logarithmic capacity. This is not clear even for the case of $\operatorname{Dir}(\mathbb{D})$. We require some preliminaries.

Theorem 5.6 (Brown and Shields [BrSh]). If $f \in \operatorname{Dir}(\mathbb{D})$ and $Z(f)=\left\{e^{i \theta}\right.$ : $\lim f\left(r e^{i \theta}\right)=0$ as $\left.r \uparrow 1\right\}$ has positive logarithmic capacity, then $f$ is not cyclic for $\left(M_{z}, \operatorname{Dir}(\mathbb{D})\right)$.

Corollary 5.7. If $f \in \operatorname{Dir}(\mathscr{R})$ and $Z(f)=\left\{e^{i \theta}: \lim f\left(r e^{i \theta}\right)=0\right.$ as $\left.r \downarrow R\right\}$ has positive logarithmic capacity, then $f$ is not cyclic for $\left(M_{1 / z}, \operatorname{Dir}(\mathscr{R})\right)$.

Before we consider the case of a general bounded finitely connected circular region, we will take a look at a simpler case for the annulus $\mathscr{A}$.

Lemma 5.8. If $f \in \operatorname{Dir}(\mathscr{A})$, and if $Z_{0}(f) \cup Z_{1}(f)$ has positive logarithmic capacity, then $f$ is not cyclic.

Proof. We only prove the case $Z_{0}(f)$ has positive logarithmic capacity. We define orthogonal projections $Q_{0}, Q_{1}: \operatorname{Dir}(\mathscr{A}) \rightarrow \operatorname{Dir}(\mathscr{A})$ by $Q_{0}(h)=h_{0}$, and $Q_{1}(h)=h_{1}$ where $h=h_{0}+h_{1}, h_{0} \in \operatorname{Dir}(\mathbb{D})$ and $h_{1} \in \operatorname{Dir}(\mathscr{R})$.

Let $J_{n}=\left\{e^{i \theta} \in Z_{0}(f):\left|f_{0}\left(r e^{i \theta}\right)\right| \leq n, R<r<1\right\}$. These are Borel sets and $Z_{0}(f)=\bigcup_{n=1}^{\infty} J_{n}$. There exists an integer $M$ such that $J_{M}$ has a positive capacity. Hence there is a compact set $F \subset J_{M}$ and a Borel probability measure $\mu$ supported on $F$, with finite energy integral. This means that

$$
I(\mu)=\iint \log \frac{1}{|z-w|} d \mu(z) d \mu(w)<\infty .
$$

It is shown in the proof of Theorem 5 of $[\mathrm{BrSh}]$ that the linear functional $\lambda: \operatorname{Dir}(\mathbb{D}) \rightarrow \mathbb{C}$ defined by $\lambda(h)=\lim _{r \uparrow 1} \int_{F} h(r w) d \mu(w)$ is continuous.

Define linear functionals $\alpha, \beta: \operatorname{Dir}(\mathscr{A}) \rightarrow \mathbb{C}$ by

$$
\alpha(h)=\lim _{r \uparrow 1} \int_{F}\left(Q_{0} h\right)(r w) d \mu(w), \quad \text { and } \quad \beta(h)=\int_{F}\left(Q_{1} h\right)(w) d \mu(w) .
$$

In fact $\alpha=\lambda \circ Q_{0}$ and so it is continuous. One might use Lemma 2.1 to show that $\beta$ is continuous. Let $p$ be a polynomial in $z$ and $1 / z$, we have,

$$
(\alpha+\beta)(p f)=\lim _{r \uparrow 1} \int_{F}(p f)(r w) d \mu(w) .
$$

Notice that $Q_{0}(p f)=Q_{0}\left(p f_{0}+p f_{1}\right)=p f_{0}+q$ where $q$ is a polynomial in $z$ and $1 / z$. So $Q_{0}(p f)(r w)$ is bounded for $w \in J_{M}$ and $R<r<1$. By the bounded convergence theorem, $(\alpha+\beta)(f)=0$ and hence $f$ is not cyclic. 
Theorem 5.9. If $f \in \operatorname{Dir}(\Omega)$ and one of the sets $Z_{0}(f), Z_{1}(f), \ldots, Z_{N}(f)$ has positive logarithmic capacity, then $f$ is not cyclic.

Proof. We only consider the case $Z_{0}(f)$ has positive logarithmic capacity. Note that the restriction of $f$ on $A_{0}$ is a function in $\operatorname{Dir}\left(A_{0}\right)$ and is not cyclic in $\operatorname{Dir}\left(\mathscr{A}_{0}\right)$ by the last lemma. One can show that the constant function 1 does not lie in $[f]$ and hence $f$ is not cyclic.

\section{INVARIANT SUBSPACES}

In what follows, by invariant subspace, we always mean a closed subspace of $\operatorname{Dir}(\Omega)$ which is invariant under all multiplication operators.

If $\mathscr{M}$ is an invariant subspace of $\operatorname{Dir}(\Omega)$ and $f \in \mathscr{M}$, then $[f] \subset \mathscr{M}$. Let $\xi$ be a fixed point in $\Omega$ and $\mathscr{M}=\{f \in \operatorname{Dir}(\Omega): f(\xi)=0\}$. Being the kernel of a continuous linear functional, $\mathscr{M}$ is closed. It is obvious that $\mathscr{M}$ is invariant. As we shall see later, every invariant subspace of codimension 1 is of this form. We need two lemmas whose proofs are straightforward and are omitted.

Lemma 6.1. Let $\mathscr{M}$ be a closed finite codimensional subspace of a Banach space $\mathscr{X}$. Let $T: \mathscr{X} \rightarrow \mathscr{X}$ be an invertible bounded linear operator. We have the following.

(1) If $T \mathscr{M} \subset \mathscr{M}$, then $T^{-1} \mathscr{M} \subset \mathscr{M}$.

(2) If $T \mathscr{M} \subset \mathscr{M}$, then $T \mathscr{M}=\mathscr{M}$.

Lemma 6.2. If $p(z)=\left(z-\alpha_{1}\right) \cdots\left(z-\alpha_{k}\right)$ is a polynomial where $\alpha_{i} \in \Omega$, then

(1) $p \operatorname{Dir}(\Omega)=\left\{f \in \operatorname{Dir}(\Omega): f\left(\alpha_{1}\right)=\cdots=f\left(\alpha_{k}\right)=0\right\}$ where in case of zeros of $p$ of multiplicity larger than one, we require derivatives of appropriate orders to equal zero.

(2) $\operatorname{dim} \operatorname{Dir}(\Omega) / p \operatorname{Dir}(\Omega)=\operatorname{deg} p=k$.

The following result is a complete characterization of all finite codimensional invariant subspaces of $\operatorname{Dir}(\Omega)$. A similar characterization for Bergman spaces was obtained by Axler and Bourdon in [AxBo].

Theorem 6.3. Let $\mathscr{M}$ be a finite codimensional closed subspace of $\operatorname{Dir}(\Omega)$. The following are equivalent.

(1) $z \mathscr{M} \subseteq \mathscr{M}$.

(2) $(z-a)^{-1} \mathscr{M} \subset \mathscr{M}$ for some $a \notin \Omega^{-}$.

(3) $(z-a)^{-1} \mathscr{M}=\mathscr{M}$ for some $a \notin \Omega^{-}$.

(4) $(z-a)^{-1} \mathscr{M} \subset \mathscr{M}$ for all $a \notin \Omega^{-}$.

(5) $(z-a)^{-1} \mathscr{M}=\mathscr{M}$ for all $a \notin \Omega^{-}$.

(6) $\phi \mathscr{M} \subseteq \mathscr{M}$ for all $\phi$ in $M(\operatorname{Dir}(\Omega))$.

(7) $\mathscr{M}=q \operatorname{Dir}(\Omega)$ where $q$ is a polynomial with all its roots lying in $\Omega$.

Proof. We need only to show (1) implies (7). The other implications are obvious. Suppose (1) is true. Define a linear operator $Y: \operatorname{Dir}(\Omega) / \mathscr{M} \rightarrow \operatorname{Dir}(\Omega) / \mathscr{M}$ by $Y(f+\mathscr{M})=z f+\mathscr{M}$. There is a nonzero polynomial $q$ with degree at most $\operatorname{dim} \operatorname{Dir}(\Omega) / \mathscr{M}$ such that $q(Y)=0$, that is $q \operatorname{Dir}(\Omega) \subset \mathscr{M}$. 
Factorize $q=q_{1} q_{2}$ where $q_{1}$ is a polynomial whose roots lie inside $\Omega$ and $q_{2}$ is a polynomial with no roots in $\Omega$. By Lemma $5.4, q_{2} \operatorname{Dir}(\Omega)$ is dense in $\operatorname{Dir}(\Omega)$. Using the fact that $q_{1}$ is a multiplier, one can verify that $q_{1} \operatorname{Dir}(\Omega) \subset\left(q_{1} q_{2} \operatorname{Dir}(\Omega)\right)^{-}=(q \operatorname{Dir}(\Omega))^{-} \subset \mathscr{M}$. By Lemma 6.2, we get

$$
\begin{array}{r}
\operatorname{dim} \operatorname{Dir}(\Omega) / \mathscr{M} \leq \operatorname{dim} \operatorname{Dir}(\Omega) / q_{1} \operatorname{Dir}(\Omega) \\
=\operatorname{deg} q_{1} \leq \operatorname{deg} q \leq \operatorname{dim} \operatorname{Dir}(\Omega) / \mathscr{M} .
\end{array}
$$

Hence $q$ is a constant multiple of $q_{1}$ and $\mathscr{M}=q \operatorname{Dir}(\Omega)$.

Although the image set of a function in a Dirichlet space has finite area (counting multiplicity), the function need not be bounded. However, it is a quotient of two bounded Dirichlet functions. To prove that we require a result of Nguyen Xuan Uy. Here, we denote $\mathbb{C}^{*}=\mathbb{C} \cup\{\infty\}$, the extended plane.

Theorem 6.4 (Nguyen Xuan Uy [Nguy]). If $K$ is a compact subset of $\mathbb{C}$ with positive area then there is a nonconstant function $g$ analytic in $\mathbb{C}^{*} \backslash K$ such that $g$ and $g^{\prime}$ and bounded.

Theorem 6.5. If $G$ is an open connected subset of $\mathbb{C}$ and $f \in \operatorname{Dir}(G)$, then there exist $g, h \in H^{\infty}(G) \cap \operatorname{Dir}(G)$ such that $f=g / h$.

Proof. The area (counting multiplicity) of the image set $f(G)$ is finite. There exists a compact set $K$ in $\mathbb{C} \backslash f(G)$ with positive area. By Theorem 6.4, there is a function $p$ analytic in $\mathbb{C}^{*} \backslash K$ such that $p, p^{\prime}$ are in $H^{\infty}(\mathbb{C} \backslash K)$. We may assume $p(\infty)=0$. The function $h(z)=p \circ f(z)$ is in $H^{\infty}(G) \cap \operatorname{Dir}(G)$. Let $g=f h$. It remains to show that $g \in H^{\infty}(G) \cap \operatorname{Dir}(G)$.

Since $p(\infty)=0$, we write, $p(z)=a_{1} z^{-1}+a_{2} z^{-2}+\cdots$ for large $|z|$. We let $w=f(z)$ and so $g(z)=w p(w)$ which is bounded in $\mathbb{C}^{*} \backslash K$. The function $w p^{\prime}(w)$ is bounded in $\mathbb{C} \backslash K$ and so $g^{\prime}=f^{\prime}(p \circ f)+f\left(p^{\prime} \circ f\right) f^{\prime}$ is in $B(G)$.

Next we want to show that each nontrivial invariant subspace contains bounded functions, and any two nontrivial invariant subspaces have a nontrivial intersection. This result suggests the possibility of a description of the invariant subspaces analogous to that obtained by Beurling for the Hardy space $H^{2}$ in the unit disk $\mathbb{D}$, and generalized by several authors to finitely connected regions. However, it is shown in $[\mathrm{BeFP}]$ that there exists a family $\left\{M_{\alpha}: \alpha \in \mathbb{C}\right\}$ of closed subspaces of $B(\mathbb{D})$ invariant under multiplication by $z$ such that $M_{\alpha} \cap M_{\beta}=\{0\}$ if $\alpha \neq \beta$.

Theorem 6.6. If $\mathscr{M}$ and $\mathscr{N}$ are two nonzero invariant subspaces of $\operatorname{Dir}(\Omega)$, then $\mathscr{M} \cap H^{\infty}(\Omega) \neq\{0\}$, and $\mathscr{M} \cap \mathscr{N} \neq\{0\}$.

Proof. We let $f$ be a nonzero function in $\mathscr{M}$ and let $f=g / h$ where $g$ and $h$ are in $\operatorname{Dir}(\Omega) \cap H^{\infty}(\Omega)$. By Theorem 4.7, $h_{t} f \rightarrow g$ as $t \uparrow 1$ and so $g \in[f]$.

We let $f_{1}, f_{2}$ be two bounded functions in $\mathscr{M}$ and $\mathscr{N}$ respectively. The product $f_{1} f_{2} \in \operatorname{Dir}(\Omega)$. As $t \uparrow 1,\left(f_{1}\right)_{t} f_{2} \rightarrow f_{1} f_{2}$. So $f_{1} f_{2} \in \mathcal{N}$, and by symmetry, $f_{1} f_{2} \in \mathscr{M}$. 


\section{TRANSITIVE OPERATOR ALgEBRAS}

Let $X$ be a Banach space and $\mathscr{L}(X)$ be the algebra of all bounded operators on $X$. An operator subalgebra (containing the identity) $\mathscr{C}$ of $\mathscr{L}(X)$ is said to be a transitive algebra if there is no nontrivial closed subspace of $X$ invariant under every operator of $\mathscr{C}$. Let $k$ be a positive integer. An algebra $\mathscr{C} \subset$ $\mathscr{L}(X)$ is said to be $k$-fold transitive if for any choice of elements $x_{1}, \ldots, x_{k}$, $y_{1}, \ldots, y_{k}$ in $X$ with $x_{1}, \ldots, x_{k}$ linearly independent, there exists a sequence $\left\{A_{j}\right\}_{1}^{\infty} \subset \mathscr{C}$ such that for $1 \leq i \leq k$,

$$
\lim A_{j} x_{i}=y_{i} \text { as } j \rightarrow \infty .
$$

In fact $\mathscr{C}$ is transitive if and only if $\mathscr{C}$ is 1 -fold transitive. In [Arve] Arveson remarked that if $\mathscr{C}$ is $k$-fold transitive for all positive integers $k$ then $\mathscr{C}$ is SOT dense in $\mathscr{L}(X)$. In the same paper he proved that in case $X$ is a Hilbert space, every transitive operator algebra containing the unilateral shift must be SOT dense in $\mathscr{L}(X)$.

By $X^{(k)}$, we denote the Banach space $X \oplus \cdots \oplus X$ ( $k$ copies) consisting of vectors of the form $\left\langle x_{1}, \ldots, x_{k}\right\rangle, x_{i} \in X$. Let $U_{i}: \mathfrak{D}_{i} \rightarrow X \quad(i=1,2)$ be linear transformations defined on a vector subspace $\mathfrak{D}_{i}$ of $X$. We write $U_{1} \subset U_{2}$ if $\Gamma\left(U_{1}\right) \subset \Gamma\left(U_{2}\right)$ where $\Gamma\left(U_{i}\right)$ denotes the graph of $U_{i}$. If $\mathscr{C}$ is a subalgebra of $\mathscr{L}(X)$ and $U: \mathfrak{D} \rightarrow X$ is a linear transformation, we say that $U$ commutes with $\mathscr{C}$ if for every $B$ in $\mathscr{C}, B U \subset U B$.

Theorem 7.1 (Arveson [Arve]). Let $X$ be a Banach space and $\mathscr{C}$ be a transitive subalgebra of $\mathscr{L}(X)$. Then $\mathscr{C}$ is not 2-fold transitive if and only if there exists a closed densely defined, nonscalar linear transformation that commutes with $\mathscr{C}$.

Let $k \geq 2$, and suppose $\mathscr{C}$ is $k$-fold transitive but not $(k+1)$-fold transitive. Then there exist $k$ linear transformations $T_{1}, \ldots, T_{k}$ defined on a common domain $\mathfrak{D}$, such that

(1) each $T_{j}$ commutes with $\mathscr{C}$,

(2) no $T_{j}$ is closable,

(3) $\left\{\left\langle x, T_{1} x, \ldots, T_{k} x\right\rangle: x \in \mathfrak{D}\right\}$ is a closed subspace of $X^{(k+1)}$.

We shall prove that if $\mathscr{C} \subset \mathscr{L}(\operatorname{Dir}(\Omega))$ is a transitive operator algebra and $\mathscr{C}$ contains all the multiplication operators then $\mathscr{C}$ is SOT dense in $\mathscr{L}(\operatorname{Dir}(\Omega))$. We need to show that $\mathscr{C}$ is $k$-fold transitive for all $k \geq 2$. If $\psi$ is a function defined on $\Omega$, we define the vector subspace $\mathfrak{D}\left(M_{\psi}\right)=\{f \in \operatorname{Dir}(\Omega): \psi f \in$ $\operatorname{Dir}(\Omega)\}$ to be the domain of the linear transformation of multiplication by $\psi$. In fact $\psi \in M(\operatorname{Dir}(\Omega))$ if and only if $\mathfrak{D}\left(M_{\psi}\right)=\operatorname{Dir}(\Omega)$.

Lemma 7.2. Let $\mathscr{C}$ be a subalgebra of $\mathscr{L}(\operatorname{Dir}(\Omega))$ containing $\left\{M_{\phi}: \phi \in\right.$ $M(\operatorname{Dir}(\Omega))\}$ and let $T_{1}, \ldots T_{k}: \mathfrak{D} \rightarrow \operatorname{Dir}(\Omega)$ be $k$ linear transformations defined on the vector subspace $\mathfrak{D}$ of $\operatorname{Dir}(\Omega)$. Suppose each $T_{j}$ commutes with $\mathscr{C}$ and $\mathfrak{M}=\left\{\left\langle f, T_{1} f, \ldots, T_{k} f\right\rangle: f \in \mathfrak{D}\right\}$ is closed in $\operatorname{Dir}(\Omega)^{(k+1)}$. Then there exist $u_{i}$ and $v_{i}$ in $H^{\infty}(\Omega) \cap \operatorname{Dir}(\Omega)$ such that $T_{i} \subset M_{\phi_{t}}$, where $\phi_{i}=u_{i} / v_{i}$ $(1 \leq i \leq k)$. 
Proof. We shall show that for all $f, h$ in $\mathfrak{D}, T_{j} f / f=T_{j} h / h \quad(1 \leq j \leq k)$, and hence if we let $\phi_{j}=T_{j} f / f$ then $\phi_{j}$ is independent of $f$ and $T_{j} f=\phi_{j} f$. By Theorem 6.5, $\phi_{j}$ is a quotient of functions in $H^{\infty}(\Omega) \cap \operatorname{Dir}(\Omega)$.

$$
\begin{array}{llll}
f=\frac{f_{1,0}}{f_{2,0}}, \quad \text { and } & T_{j} f=\frac{f_{1, j}}{f_{2, j}} & (1 \leq j \leq k), \\
h=\frac{h_{1,0}}{h_{2,0}}, \quad \text { and } & T_{j} h=\frac{h_{1, j}}{h_{2, j}} & (1 \leq j \leq k),
\end{array}
$$

where $f_{1, j}, f_{2, j}$ and $h_{1, j}, h_{2, j}$ are in $H^{\infty}(\Omega) \cap \operatorname{Dir}(\Omega)$. We let

$$
\xi=f_{2,0} f_{2,1} \cdots f_{2, k} h_{1,0} h_{2,1} \cdots h_{2, k},
$$

and

$$
\eta=f_{1,0} f_{2,1} \cdots f_{2, k} h_{2,0} h_{2,1} \cdots h_{2, k} .
$$

Note that $\xi, \eta \in \operatorname{Dir}(\Omega)$ and $\xi f=\eta h$. We shall show that $T_{j}(\xi f) / \xi f=$ $T_{j} f / f$, and by symmetry, $T_{j}(\eta h) / \eta h=T_{j} h / h$, and hence $T_{j} f / f=T_{j} h / h$. The functions $\xi f, \xi T_{1} f, \ldots, \xi T_{k} f$ are in $H^{\infty}(\Omega) \cap \operatorname{Dir}(\Omega)$. By hypothesis,

$$
\left\langle\xi_{t} f, T_{1}\left(\xi_{t} f\right), \ldots, T_{k}\left(\xi_{t} f\right)\right\rangle=\left\langle\xi_{t} f, \xi_{t}\left(T_{1} f\right), \ldots, \xi_{t}\left(T_{k} f\right)\right\rangle
$$

is in $\mathfrak{M}$. As $t \uparrow 1$, it tends to $\left\langle\xi f, \xi\left(T_{1} f\right), \ldots, \xi\left(T_{k} f\right)\right\rangle$. Since $\mathfrak{M}$ is closed in $\operatorname{Dir}(\Omega)^{(k+1)}, \xi f \in \mathfrak{D}$ and $\xi\left(T_{j} f\right)=T_{j}(\xi f)$.

Theorem 7.3. If $\mathscr{C} \subset \mathscr{L}(\operatorname{Dir}(\Omega))$ is a transitive operator algebra and $\mathscr{C}$ contains $M_{z}, M_{1 /\left(z-\zeta_{1}\right)}, \ldots, M_{1 /\left(z-\zeta_{N}\right)}$, then $\mathscr{C}$ is SOT dense in $\mathscr{L}(\operatorname{Dir}(\Omega))$.

Proof. We may assume that $\mathscr{C}$ contains all multiplication operators by Theorem 4.8. We first show that $\mathscr{C}$ is 2 -fold transitive. Let $U: \mathfrak{D}(U) \rightarrow \operatorname{Dir}(\Omega)$ be a closed densely defined linear transformation that commutes with $\mathscr{C}$. Applying Lemma 7.2 with $k=1, \mathfrak{D}=\mathfrak{D}(U), \mathfrak{M}=\Gamma(U)$, we conclude that $U \subset M_{\phi}$ for some $\phi$ meromorphic in $\Omega$. We shall show that $\phi=c$.

For each $w$ in $\Omega$ there exists $k_{w}$ in $\operatorname{Dir}(\Omega)$ such that $f(w)=\left(f, k_{w}\right)$ for all $f$ in $\operatorname{Dir}(\Omega)$ where $(\cdot, \cdot)$ is the inner product. If $f \in \mathfrak{D}\left(M_{\phi}\right)$, then $\left(M_{\phi} f, k_{w}\right)=\left(f, \overline{\phi(w)} k_{w}\right)$. So $k_{w}$ is in the domain of $M_{\phi}^{*}$, and $M_{\phi}^{*} k_{w}=$ $\overline{\phi(w)} k_{w}$. Since $U \subset M_{\phi}, M_{\phi}^{*} \subset U^{*}$ and $U^{*} k_{w}=M_{\phi}^{*} k_{w}=\overline{\phi(w)} k_{w}$.

For $A \in \mathscr{C}, A^{*} U^{*} \subset U^{*} A^{*}$ and $U^{*} A^{*} k_{w}=A^{*} U^{*} k_{w}=\overline{\phi(w)} A^{*} k_{w}$. The algebra $\mathscr{C}^{*}=\left\{A^{*}: A \in \mathscr{C}\right\}$ is transitive. Thus $\mathscr{C}^{*} k_{w}$ is dense in $\operatorname{Dir}(\Omega)$. The adjoint operator $U^{*}$ is closed and densely defined. For $g$ in $\operatorname{Dir}(\Omega)$ there exist $g_{i}=A_{i}^{*} k_{w} \in \mathscr{C}^{*} k_{w},(i \geq 1)$ such that $g_{i} \rightarrow g$ in $\operatorname{Dir}(\Omega)$. We observe that

$$
\left\langle g_{i}, U^{*} g_{i}\right\rangle=\left\langle g_{i}, U^{*} A_{i}^{*} k_{w}\right\rangle=\left\langle g_{i}, \overline{\phi(w)} A_{i}^{*} k_{w}\right\rangle=\left\langle g_{i}, \overline{\phi(w)} g_{i}\right\rangle,
$$

which converges to $\langle g, \overline{\phi(w)} g\rangle$. But $U^{*}$ has a closed graph, so $U^{*} g=\overline{\phi(w)} g$. We conclude that $\phi$ is a constant. 
Next we show that $\mathscr{C}$ is $k$-fold transitive for $k \geq 3$. Suppose $U_{1}, \ldots, U_{k}$ : $\mathfrak{D} \rightarrow \operatorname{Dir}(\Omega)$ are $k$ linear transformations defined on a common domain $\mathfrak{D} \subset \operatorname{Dir}(\Omega)$ and each $U_{i}$ commutes with $\mathscr{C}$. We further assume that $\mathfrak{M}=$ $\left\{\left\langle f, U_{1} f, \ldots, U_{k} f\right\rangle: f \in \mathfrak{D}\right\}$ is closed in $\operatorname{Dir}(\Omega)^{(k+1)}$.

By Lemma 7.2, there exist $\phi_{i}(1 \leq i \leq k)$ meromorphic in $\Omega$ such that $T_{i} \subset M_{\phi_{i}}$. We shall show that $T_{1}$ is closable. Let $\left\{f_{m}\right\}$ be a sequence in $\mathfrak{D}\left(M_{\phi_{1}}\right)$, and $f, g$ be two functions in $\operatorname{Dir}(\Omega)$ satisfying $\left\langle f_{m}, \phi_{1} f_{m}\right\rangle \rightarrow\langle f, g\rangle$ in $\operatorname{Dir}(\Omega)^{(k+1)}$. Hence for each $z$ in $\Omega, g(z)=\phi_{1}(z) f(z)$. That is $g=$ $M_{\phi_{1}} f$.

\section{REFERENCES}

[Arve] W. B. Arveson, A density for operator algebras, Duke Math. J. 34 (1967), 635-647.

[AxBo] S. Axler and P. Bourdon, Finite codimensional invariant subspaces of Bergman spaces. preprint, 1986.

[AxSh] S. Axler and A. L. Shields, Univalent multipliers of the Dirichlet space, Michigan Math. J. 32 (1985), 65-80.

[BeFP] H. Bercovici, C. Foias and C. M. Pearcy, Dual algebras with applications to invariant subspaces and dilation theory, CBMS Regional Conf. Ser. in Math., no. 56, Amer. Math. Soc., Providence, R.I., 1985.

[BrSh] L. Brown and A. L. Shields, Cyclic vectors in the Dirichlet space, Trans. Amer. Math. Soc. 285 (1984), 269-304.

[ChPS] B. Chevreau, C. M. Pearcy and A. L. Shields, Finitely connected domains $G$, representations of $H^{\infty}(G)$, and invariant subspaces, J. Operator Theory 6 (1981), 375-405.

[DuRS] P. L. Duren, B. W. Romberg, and A. L. Shields, Linear functionals on $H^{p}$ spaces with $0<p<1$, J. Reine Angew. Math. 288 (1969), 32-60.

[Nguy] Nguyen Xuan Uy, Removable sets of analytic functions satisfying a Lipschitz condition, Ark. Math. 17 (1979), 19-27, MR 81i:30076.

[Shie] A. L. Shields, Cyclic vectors in some spaces of analytic functions, Proc. Roy. Irish Acad. Sect. A 74 (1974), 293-296.

Department of Mathematics, University of Michigan, Ann Arbor, Michigan 48109

Current address: Department of Mathematics, Michigan State University, East Lansing, Michigan 48824-1027 